\title{
Les lésions du système nerveux central au cours du SIDA
}

Au moins $75 \%$ des examens post-mortem de cas de SIDA révèlent des lésions du système nerveux central qui peuvent être classées en infections opportunistes (parasitaires, mycotiques et surtout virales), en conséquences directes de l'infection par le virus du SIDA et en tumeurs (principalement des lymphomes primitifs développés aux dépens de lymphocytes $B$ ). La fréquence des atteintes qui peuvent être directement en relation avec l'infection par le virus du SIDA, caractérisées notamment par la présence de cellules géantes mononucléées, augmente actuellement. Dans la plupart des observations, les lésions neurologiques sont en réalité la conséquence de processus intriqués, expliquant l'aspect atypique et polymorphe de la symptomatologie.

\section{Françoise Gray}

\begin{abstract}
ADRESSE
F. Gray : maître de conférence des universités, praticien hospitalier. Département de pathologie (neuropathologie), hôpital Henri-Mondor, Créteil. Département de neurosciences médicales et département de pathologie cel lulaire et tissulaire, faculté de médecine de Créteil, université Paris-Val-de-Marne. Hôpital Henri-Mondor, 94010 Créteil Cedex, France.
\end{abstract}

\section{TIRÉS A PART}

F. Gray. es atteintes du système nerveux central (SNC) en rapport avec une infection par le virus de l'immunodéficience humaine (HIV) (human immune deficiency virus), contrairement aux atteintes du système nerveux périphérique, surviennent, le plus souvent, au cours de syndromes d'immunodépression acquise (SIDA) confirmés ; d'authentiques encéphalites à HIV révélatrices de la maladie ont été cependant exceptionnellement rapportées [12]. Ces atteintes sont graves, souvent responsables du décès des malades et fréquentes. Des signes cliniques d'atteinte nerveuse centrale sont observés dans environ un tiers des cas de SIDA, des lésions du SNC sont présentes dans plus de $75 \%$ des cas autopsiés, elles seraient même constantes dans certaines études systématiques. Les lésions du SNC au cours du SIDA sont multiples et répondent à des mécanismes divers qui ne sont pas tous parfaitement élucidés [1, 2]. La baisse des défenses immunitaires à médiation cellulaire, secondaire au déficit en lymphocytes auxilliaires (T4), caractéristique de la maladie, favorise la survenue d'infections opportunistes et/ou de lymphomes non hodgkiniens. Le HIV a aussi un tropisme pour les histiocytes-macrophages porteurs du même antigène
CD4 que les lymphocytes T4, et peut infecter directement le SNC par l'intermédiaire de ces cellules. Enfin les altérations du SNC peuvent être secondaires à d'autres complications du SIDA, vasculaires ou viscérales, ou aux conditions terminales.

\section{Les infections} opportunistes

Ces infections sont en rapport avec la déficience des mécanismes de défense immunitaire à médiation cellulaire et sont très fréquentes [1, 2]. Il peut s'agir de parasitoses au premier rang desquelles la toxoplasmose cérébrale représente encore la plus fréquente des complications neurologiques du SIDA en France. Les lésions caractéristiques sont focales, souvent multiples (figure 1a) ; ce sont des abcès comportant une nécrose centrale d'allure ischémique, entourée d'une réaction granulomateuse. Dans les cas traités, ces lésions peuvent se déterger voire disparaître. Certaines formes atypiques semblent plus particulières au SIDA. La constatation d'un semis de kystes dans un parenchyme cérébral normal par ailleurs (figure $1 b$ ), sans traduction clinique n'est pas rare. Dans quelques cas, des lésions diffuses, non nécrotiques, «encéphalitiques », faites d'une dissémination de 


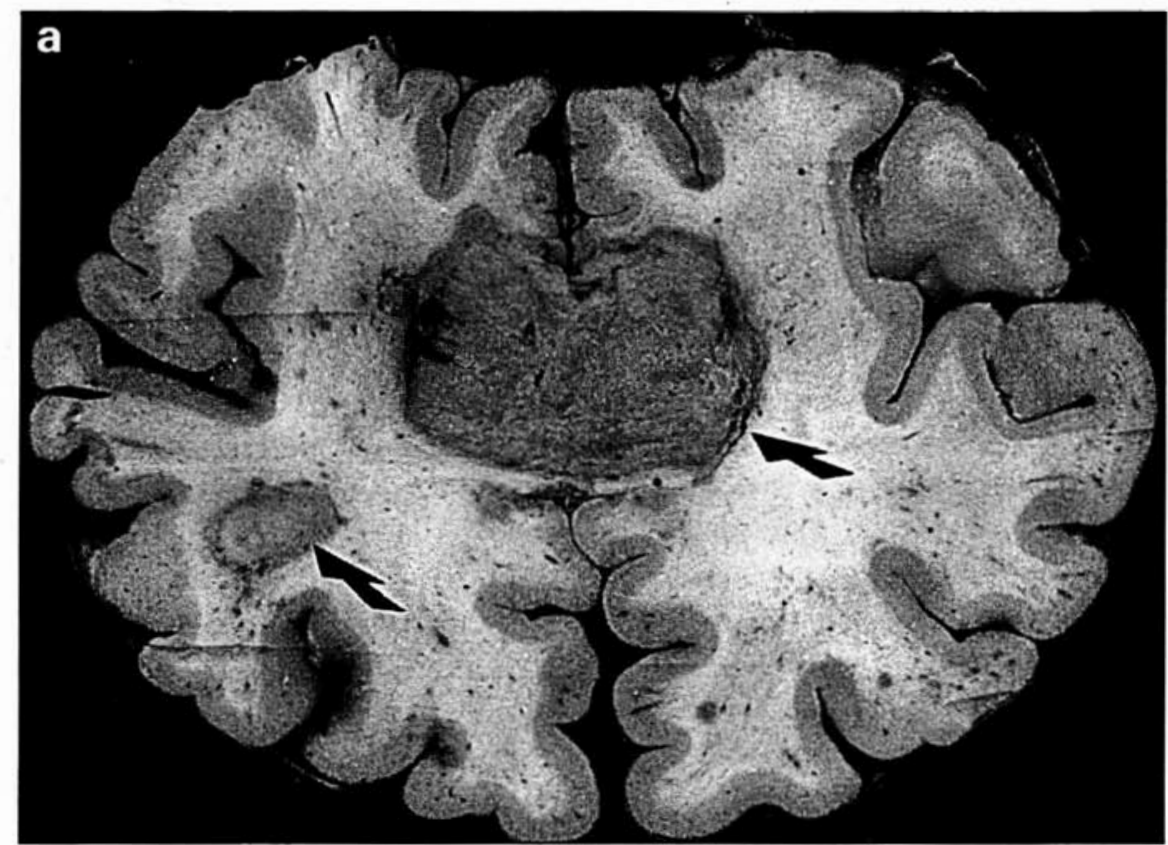

nodules microgliaux contenant des parasites sous forme libre ou enkystée ont été observées; ces formes trompeuses, car sans traduction tomodensitométrique, représentent une cause curable, presque toujours méconnue, d'encéphalopathie aiguë. Les mycoses du SNC sont, au cours du SIDA, beaucoup moins fréquentes, que la toxoplasmose, ce qui contraste avec leur incidence sans cesse croissante dans d'autres catégories d'immunodéprimés chez qui la toxoplasmose est plus rare. La cryptococcose est l'infection mycotique la moins rare au cours du SIDA (figure 2). Elle détermine habituellement une méningite ou une méningoencéphalite. Les lésions méningées peuvent être diffuses ou prédominer à la base du cerveau; une diffusion intraventriculaire est possible. Les lésions intraparenchymateuses sont plus rares; la présence de kystes ne contenant que des cryptocoques, sans réaction cellulaire inflammatoire ou gliale, serait assez particulière à ce terrain. La mise en évidence du champignon dans le liquide céphalo-rachidien permet le diagnostic. D'autres infections mycotiques ont été observées : candidose, aspergillose, nocardiose, coccidioïdomycose, histoplasmose, etc. Elles réalisent des méningites, des méningo- abcès. Leur diagnostic, souvent difficile, ne peut être affirmé que par la mise en évidence du champignon. infections à pyogènes banals sont relativement rares. De rares cas de listériose [3] et quelques observations de syphilis cérébroméningée ou de tabès aigu ont été décrits. Les infections à mycobactéries sont plus caractéristiques. Quelques cas d'infection tuberculeuse, méningites, abcès d'installation aiguë, ou tuberculomes plus chroniques, ont été observés, surtout chez les Haïtiens et les toxicomanes. Les infections à mycobactéries atypiques sont fréquentes et le plus souvent asymptomatiques. Le germe le plus fréquemment en cause, aux Etats-Unis, est Mycobacterium avium intracellulare. Ce micro-organisme peut être mis en évidence dans le cerveau de malades porteurs d'infection systémique, à l'intérieur de macrophages périvasculaires ou au voisinage de lésions d'autre nature.

Les infections virales sont graves et sont observées avec une fréquence croissante.

- Les infections à cytomégalovirus (CMV) sont fréquentes (environ $20 \%$ des cas). Elles déterminent des encéphalites de topographie volontiers périventriculaire. Les cellules épendymaires contenant les inclusions
Parmi les infections bactériennes, les

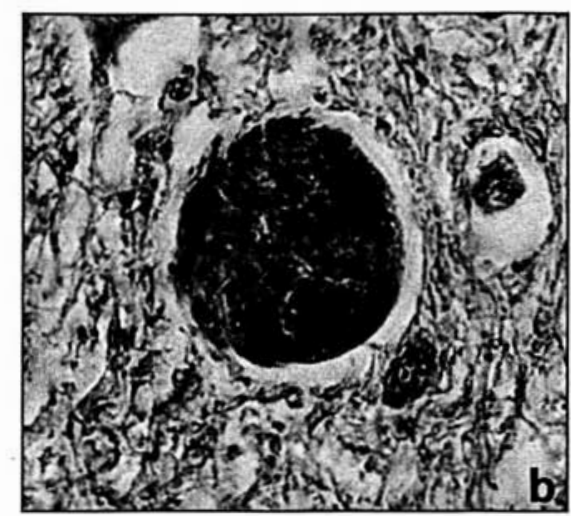

Figure 1. Toxoplasmose cérébrale. (a). Coupe vertico-frontale passant par le bec du corps calleux : présence d'abcès multiples (flèches). (b). Kyste toxoplasmique libre dans le parenchyme cérébral sans réaction inflammatoire ou gliale (hématéine-éosine, $\times 1500$ ).

caractéristiques «en œeil d'oiseau» (figure 3) semblent parfois desquamer dans la lumière ventriculaire. Dans quelques cas, l'association de lésions médullaires et radiculaires intrathécales nécrotiques et inflammatoires, avec de nombreuses inclusions virales dans les méningocytes et les cellules de Schwann, a fait évoquer la possibilité d'une dissémination par voie liquidienne, à partir d'une infection périventriculaire à CMV, à l'origine de certains tableaux de myélo-radiculite aiguë [4].

Des cas d'infection du SNC par un papovavirus réalisant une leucoencéphalopathie multifocale progressive (LEMP) sont observés avec une fréquence croissante. Les lésions démyélinisantes, en petits foyers confluents, siègent avec prédilection dans la substance blanche sous corticale (figure 4). L'examen microscopique montre des astrocytes volumineux, bizarres, parfois monstrueux et des oligodendrocytes transformés, également de grande taille, contenant des inclusions virales intranucléaires caractéristiques. Au cours du SIDA, les lésions sont souvent particulières par leur extension, leur topographie asymétrique, l'atteinte fréquente du lobe temporal, du cervelet et du tronc cérébral, et l'abondance des inclusions virales.

- Les infections par l'herpès sim- 
plex (HSV) sont relativement rares sur ce terrain et sont dues dans les rares cas rapportés à HSV de type 2 . - Une leucoencéphalite multifocale en rapport avec le virus de la varicelle et du zona a été rapportée dans quelques cas; les lésions sont semblables à celles observées chez des immunodéprimés d'autre origine, une atteinte artéritique étant possible.

- Une exceptionnelle infection par une algue (Prototheca zopfii) a même été rapportée.

\section{Les tumeurs cérébrales}

La survenue de lymphomes nonhodgkiniens au cours du SIDA n'est pas rare. Elle serait aussi, probable-

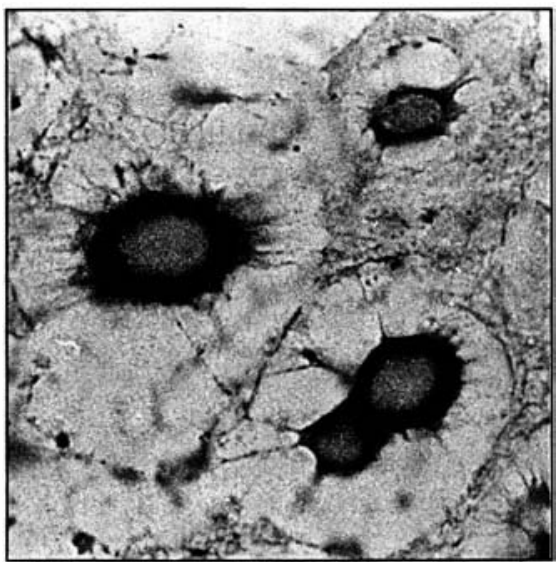

Figure 2. Cryptococcose. Présence de multiples champignons dans les leptoméninges (bleu Alcian, $\times 1500$ ).

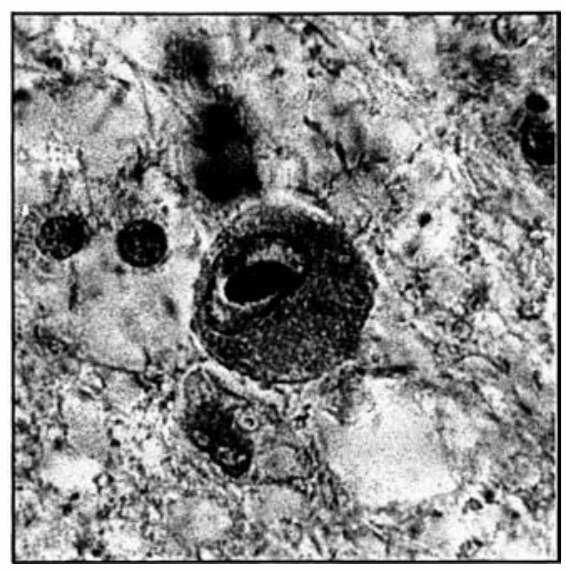

Figure 3. Encéphalite à CMV. Inclusion intranucleaire caractéristique *en œil d'oiseau * (hématéine-éosine $x$ 1500).

$\mathrm{m} / \mathrm{s}$ no 3 vol. 5 , mars 89 ment, la conséquence du déficit en lymphocytes $T$ auxilliaires (T4). Ces lymphomes sont particuliers par leur topographie extranodale prédominante et leur haute malignité. La constatation de lymphomes de Burkitt vrais au cours du SIDA a un intérêt étiopathogénique ; en effet, certains de ces lymphomes ont pu être rapportés à une infection persistante par le virus d'Epstein Barr (EBV), et il a été démontré que tous les malades atteints de SIDA ont une sérologie positive pour l'EBV*. Les lymphomes primitifs du SNC représentent la localisation la plus fréquente des lymphomes extranodaux au cours du SIDA ; ils sont particulièrement graves. Ce sont toujours des lymphomes immunoblastiques à grandes cellules. Dans tous les cas où la prolifération a été typée, il s'agissait d'un lymphome de type B. Des localisations secondaires de lymphomes ou de plasmocytomes viscéraux généralisés peuvent intéresser le SNC. Ces tumeurs siègent souvent au niveau des espaces sous-arachnoïdiens, aussi sont-elles plus souvent détectées par l'examen du liquide céphalo-rachidien que les lymphomes primitifs.

Les localisations cérébrales de sarcome de Kaposi sont rares, compte tenu de la fréquence des localisations viscérales multifocales de ce sarcome. Les rares tumeurs observées correspondent probablement à des métastases et ont toujours observées associées à des localisations pulmonaires.

\section{Infection directe du SNC par le HIV}

La preuve de l'infestation directe du SNC par le HIV a été apportée par diverses techniques virologiques, immunologiques et ultrastructurales [1].

\footnotetext{
* Par analogie avec ce qui se passe pour le lymphome de Burkilt africain, on pense qu'une infection persistante par un antigène viral comme l'EBV entraîne une stimulation des lymphocytes $B$ non réprimée par les lymphocytes $T$ aboutissant tôt ou tard à une prolifération maligne. Il est du reste remarquable que, au début tout au moins, la prolifération lymphomateuse puisse être oligoclonale et qu'elle puisse être précédée par un stade de prolifération lymphoïde polyclonale.
}

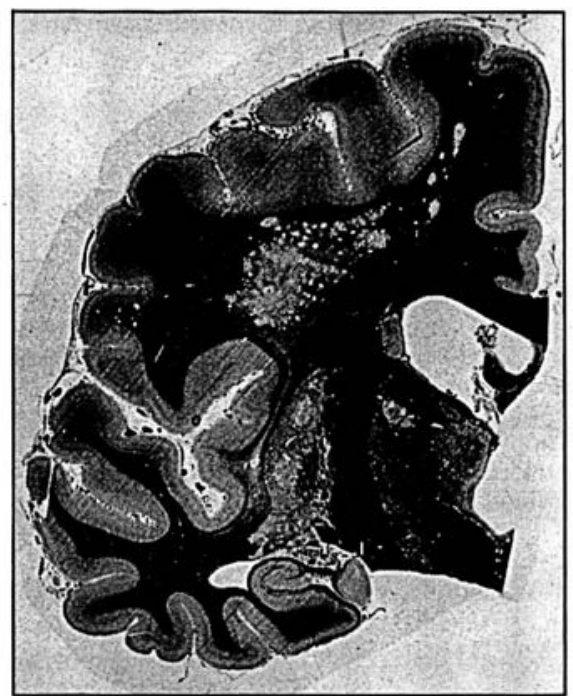

Figure 4. Leucooncéphalite multifocale progressive due à une infection par un papovavirus. Multiples foyers démyélinisés confluant dans la substance blanche pariétale sous-corticale (coupe vertico-frontale de l'hémisphère cérébral gauche passant par le noyau rouge, Loyez).

Le substratum anatomique le plus caractéristique de cette «encéphalite à HIV » est une encéphalite subaiguë avec des cellules géantes multinucléées (CGM) très particulières et que la plupart des auteurs reconnaissent comme signant l'infection du système nerveux central par le virus. Les CGM décrites pour la première fois par Sharer en 1985 [5], sont des cellules volumineuses, le plus souvent arrondies, mais parfois plus irrégulières, dont les contours cellulaires sont très nets. Leur cytoplasme éosinophile, granuleux, est souvent clair, vacuolisé à la périphérie, avec un centre plus dense. Elles contiennent plusieurs noyaux basophiles arrondis ou allongés, disposés en cercle ou en demi-cercle à la périphérie, mais qui peuvent aussi être groupés au centre ou répartis sans ordre (figure 5a). L'origine histiocytaire de ces cellules a été démontrée par les études immunohistochimiques et plusieurs études ultrastructurales ont confirmé la présence de particules virales caractéristiques du HIV dans leur cytoplasme. Ce sont des formations arrondies ou ovalaires, d'environ $100 \mathrm{~nm}$ de diamètre, entourées d'une double membrane, avec un 


\section{RÉFÉRENCES}

Une mise au point récente sur rétrovinus et système nerveux peut être trouvée dans:

1. Retrovirus in the Nervous System. Proceedings of symposium sponsored by the National Institute of Health. Bethesda, 4-6 May 1987. Ann Neurol 1988 ; 23 (suppl) : S1-217.

Une liste très complète des références concernant la neuropathologie du SIDA peut être trouvée dans :

2. Gray F, Gherardi R, Scaravilli F. The neuropathology of the acquired immune deficiency syndrome (AIDS): a review. Brain 1988 ; 111 : 245-66.

Nous ajoutons seulement les références des travaux cités dans le texte et/ou panus depuis :

3. Mascola L, Lieb L, Chiu J, Fannin SL, Linnan M. Listeriosis : an uncommon opportunistic infection in patients with acquired immudodeficiency syndrome. A report of five cases and a review of the literature. $A m \mathrm{~J} \mathrm{Med}$ $1988 ; 84: 162-4$.

4. Mahieux F, Gray F, Fénelon G, et al. Acute myeloradiculitis due to cytomegalavirus as the initial manifestation of AIDS. J Neurol Neurosurg Psychiat 1989 (sous presse).

5. Sharer LR, Cho ES, Epstein LG. Multinucleated giant cells dans HTLV-III in AIDS encephalopathy. Hum Pathol 1985; 16: 760.

6. Koenig S, Gendelman GE, Orenstein JM, et al. Detection of AIDS virus in macrophages in brain tissue from AIDS patients with encephalopathy. Science 1986; 233 : 1089-93.

7. Gray F, Gherardi R, Keohane C, Favolini M, Sobel A, Poirier J. Pathology of the central nervous system in 40 cases of acquired immune deficiency syndrome (AIDS). Neuropathol Appl Neurobiol 1988 ; 14 : 365-80.

8. Gray F, Fénelon G, Gherardi R, Favolini M, Goulon M, Guillard A. Étude neuropathologique de 15 cas d'encéphalite à cellules géantes multinucléées au cours du syndrome d'immunodéficience acquise (SIDA). $A n n$ Pathol 1988 ; 8 : 282-90.

9. Sharer LR, Epstein LG, Cho ES, Murphey-Corb M, Baskin GB, Comparative pathology of HIV and SIV encephalitides. $J$ Neuropathol Exp Neurol 1987; 46: 349 (abstr.).

10. Gray F, Gaulard P, Gherardi R, Le Bezu M, Poirier J. HIV encephalitis-like multinucleated giant cells in a nodal lymphoma in AIDS. Virchow Arch 1989 (sous presse).

11. Budka H, Costanzi G, Cristina S, et al. Brain pathology induced by infection with the human immunodeficiency virus (HIV). A histological, immunocytochemical and electron microscopical study of 100 autopsy cases.
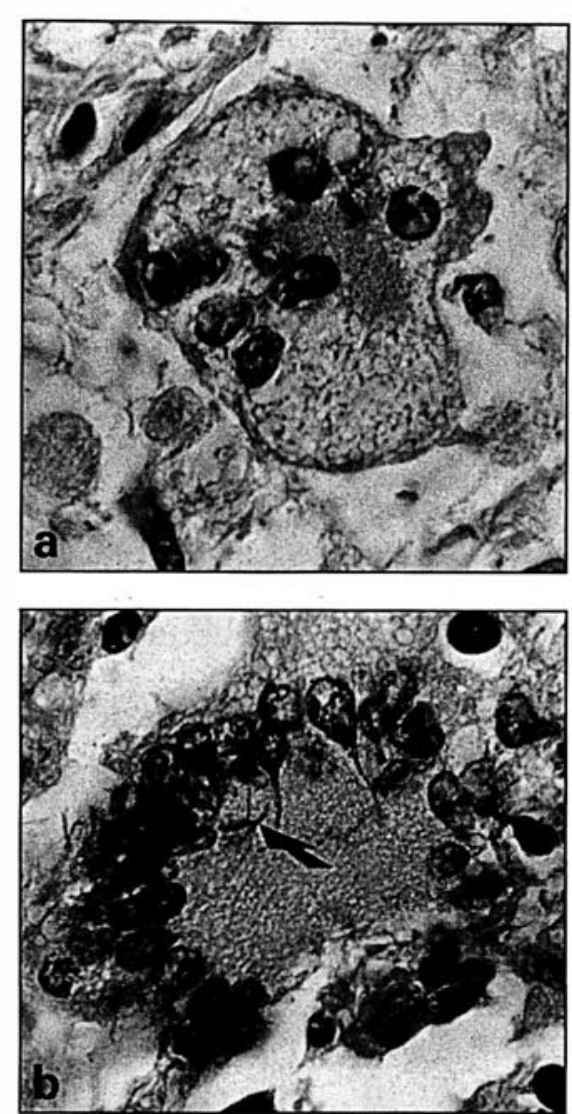

Figure 5. Collules géantes multinuclééos. (a). (Hématéine-éosine, $x$ $1500)$. (b). Cellule géante multinucléé avec des ponts nucléaires (flèche) (hématéine-éosine, $\times 1$ 600).

noyau dense aux électrons, arrondi ou étoilé, souvent excentrique, et/ou une capside centrale, moins dense, conique. Koenig et al. [6], en associant les techniques de coculture, d'hybridation in situ, l'immunocytochimie et la microscopie électronique, ont démontré que, dans le SNC, les macrophages et les CGM qui en dérivent représentaient le principal type cellulaire pouvant synthétiser de l'ARN viral et pouvaient servir de réservoir pour le virus et de véhicule pour la dissémination de l'infection virale. Aussi, actuellement, la plupart ses auteurs s'accordent pour considérer les CGM comme le marqueur morphologique spécifique de l'encéphalite à HIV ; même si d'autres cellules peuvent contenir le virus. Ces CGM semblent résulter de la fusion des macrophages sous l'influence du virus; cela a été montré en culture et en pathologie humaine $[7,8]$. Cependant des images de ponts nucléaires (figure 5b) ont aussi été décrites [7, 8], elles suggèrent que puissent aussi survenir des divisions nucléaires. Des cellules semblables ont été observées dans le foie, la rate, les ganglions lymphatiques et le tube digestif de singes infectés par le simian immunodeficiency virus (SIV) [9]; chez l'homme, en revanche, les CGM ont toujours été vues dans le SNC, à l'exception d'une observation rapportant la présence de CGM morphologiquement typiques et ayant les caractères immuno-histochimiques des histiocytes-macrophages dans une localisation ganglionnaire médiastinale de lymphome [10].

Outre la présence de CGM, l'encéphalite à HIV est caractérisée par des lésions prédominantes de la substance blanche. Au maximum est réalisée une leuco-encéphalopathie dif fuse progressive avec démyélinisation sévère de la substance blanche hémisphérique; mais les lésions peuvent être plus limitées (encéphalite multifocale à cellules géantes), en foyers dissémimés, parfois sous-corticaux. On y observe une destruction parenchymateuse, myélinique et axonale, avec des macrophages, des CGM, et une prolifération d'astrocytes volumineux parfois géants, bizarres. Il peut s'y associer une prolifération de microglie en bâtonnets et/ou des infiltrats mononucléés. Une atteinte corticale est plus rarement signalée. Cependant, Budka et al. [11], dans une série de 100 cas, ont observé dans la moitié une «poliodystrophie diffuse » caractérisée par une perte neuronale, une prolifération microgliale en bâtonnets et une gliose astrocytaire réactive. Une atteinte méningée est possible ; elle se traduit cliniquement par une méningite aseptique et, anatomiquement, par un épaississement des leptoméninges avec des cellules inflammatoires et quelques CGM. Des lésions sténosantes des artères leptoméningées $^{*}$ - responsables d'infarctus, et comportant une prolifération intimale et, inconstamment, des signes inflammatoires - ont été observées dans quelques cas associées à une méningite et/ou une encéphalite à

* Artères leptoméningées: artères à destination cérébrale passant entre la pie-mère et la convexité cérébrale. 
CGM [12, 13].

- L'incidence de cette encéphalite à HIV apparaît croissante. L'augmentation annuelle du nombre de cas d'encéphalite à CGM a été bien démontrée par Petito et al. [14]. Cette augmentation était nette dans notre série : parmi 22 cas morts avant 1987, 6 soit $27,2 \%$ avaient une encé- phalite à CGM. Parmi les 18 cas morts en 1987, il y en avait 9 , soit $50 \%[7,8]$. Cette incidence accrue est peut-être la conséquence de modifications de la thérapeutique, de la survie des malades, voire du neurotropisme du virus, mais sûrement pas d'une meilleure reconnaissance histologique puisque toutes les
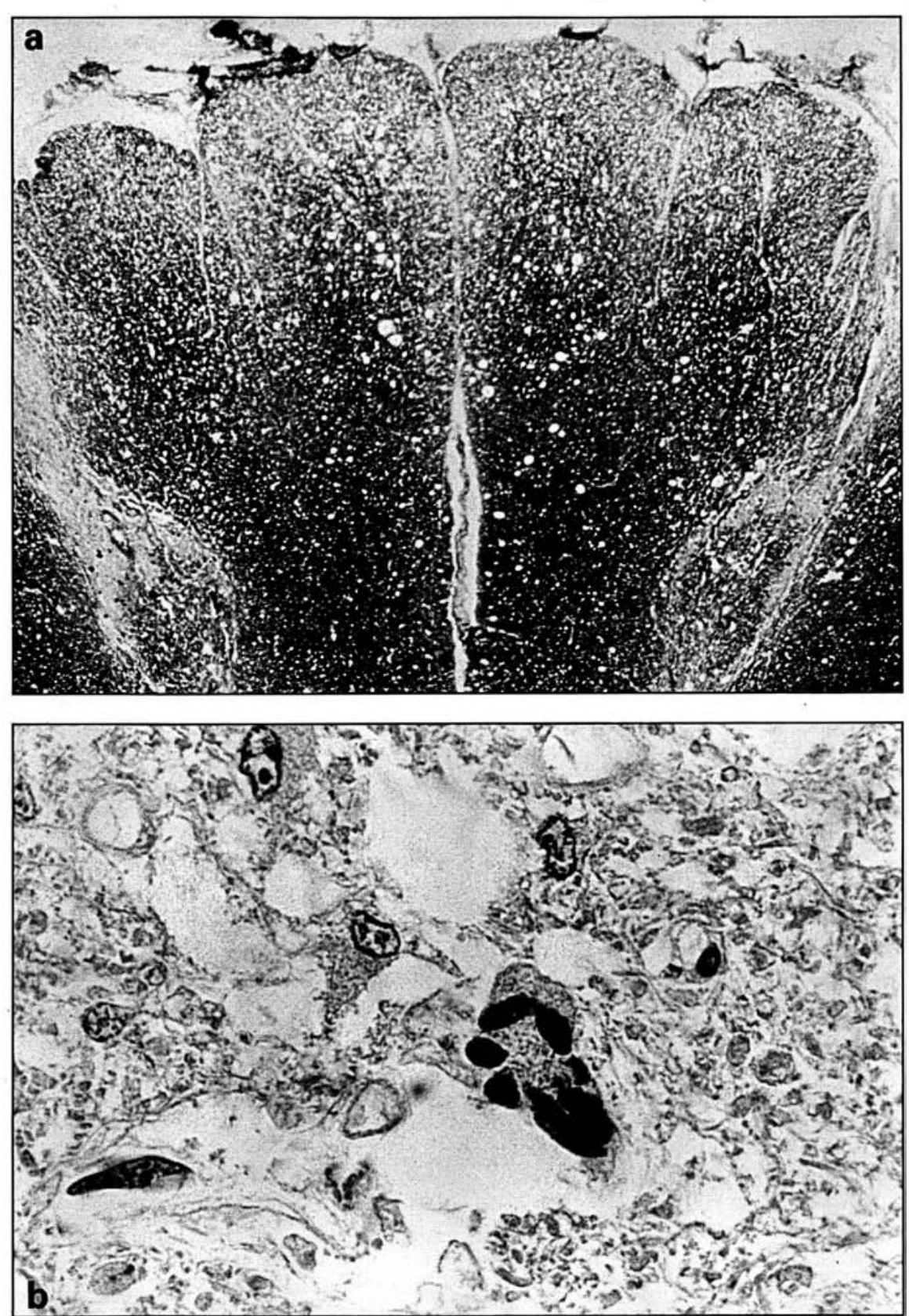

Figure 6. Myélopathie vacuolaire. (a). Lésions vacuolaires dans les cordons postérieurs (coupe horizontale de la moelle dorsale, Loyez, $\times$ 40). (b). Cellule géante multinucléée près de lésions vacuolaires (hématéine-éosine, $\times 1600$ ).

$\mathrm{m} / \mathrm{s} n^{\circ} 3$ vol. 5 , mars 89 études sont rétrospectives.

- Les corrélations anatomocliniques dans l'encéphalite à HIV sont difficiles à établir. Dans une étude anatomo-clinique de malades atteints du SIDA, déments et non déments, Navia et al. [15] ont conclu que l'encéphalite subaiguë avec CGM représentait le substratum anatomique de la démence progressive avec atrophie cérébrale caractéristique du SIDA. Outre le fait que cette entité clinique est de plus en plus discutée, une telle corrélation n'est pas toujours évidente, surtout chez l'adulte, et ce pour plusieurs raisons. D'une part, il est souvent difficile de faire la part de la détérioration intellectuelle, des troubles psychiques préexistants ou secondaires et de l'état confusionnel chez des malades souvent peu coopératifs. D'autre part, les corrélations anatomo-radiologiques ne sont pas toujours bonnes et une atrophie cérébrale observée au scanner n'est pas toujours retrouvée à l'examen post-mortem. Enfin, les troubles intellectuels sont souvent masqués par des signes focaux en rapport avec d'autres lésions associées (voir plus bas).

Certaines lésions paraissent liées à l'infection du SNC par le HIV, mais leur mécanisme reste discuté.

- Une myélopathie vacuolaire serait présente dans 0 à $30 \%$ des cas [14]. Les lésions prédominent dans les cordons postérieurs et latéraux, dans la moelle thoracique et réalisent une atteinte myélinique prédominante (figure $6 a$ ). Le mécanisme pathogénique de cette myélopathie vacuolaire reste discuté. Bien que des ressemblances existent avec la sclérose combinée, il n'a jamais été mis en évidence de déficit en vitamine $\mathrm{Bl} 2$ ou en folates. Par analogie avec la paraparésie spastique tropicale, où le rôle du virus HTLV 1 a été démontré, le rôle du HIV a été évoqué à l'origine de la myélopathie vacuolaire et plusieurs arguments soutiennent cette hypothèse: l'association fréquente de la myélopathie vacuolaire à une encéphalite à HIV, et surtout la constatation, dans quelques cas $[7 ; 8,16,17,18]$, de cellules géantes multinucléées caractéristiques de l'infection du SNC par le HIV à proximité de vacuoles médullaires (figure $6 b$ ). Cependant l'existence de myélopathies vacuolaires 
RÉFÉRENCES

12. Cho ES, Sharer LR, Peress NS, Little B. Intimal proliferation of leptomeningeal arteries and brain infarcts in subjects with AIDS. J Neurapathol Exp Neurol 1987; 46 : 385 (abstr.).

13. Scaravilli F, Daniel SE, Harcourt-Webster N, Guiloff RJ. Non-infectious vasculitis in AIDS : a possible role for HIV. Report of a case. Arch Lab Path Med 1988 (sous presse).

14. Petito CK, Cho ES, Lemann W, Navia BA, Price RW. Neuropathology of acquired immunodeficiency syndrome (AIDS): An autopsy review. J Neuropathol Exp Neurol $1986,45,635-46$.

15. Navia BA, Cho ES, Petito CK, Price RW. The AIDS dementia complex : II. Neuropathology. Ann Neurol 1986; $19: 525-35$.

16. Le Noan H, Gray F. Les atteintes médullaires au cours du syndrome d'immunodéficience acquise (SIDA) Arch Anat Cytol Path 1989 (sous presse).

17. Gray F, Gherardi R, Trotot P, Fénelon G, Poirier J. Spinal cord lesions in the acquired immune deficiency syndrome (AIDS). Neurosurg Rev. 1989 (sous presse).

18. Budka H, Maier $H$, Pohl P. Human immunodeficiency virus in vacuolar myelopathy of the acquired immune deficiency syndrome. N Engl J Med 1989 (sous presse).

19. Kamin SS, Petito CK. Vacuolar myelopathy in immunocompromised non AIDS patients. J Neuropathol Exp Neurol 1988 ; $47: 385$ (abstr.).

20. Sharer LR, Epstein LG, Cho ES, Petito CK. HTLV-III and vacuolar myelopathy. $N$ Engl J Med 1986 ; 315 : 62-3.

21. Rhodes RH. Histopathology of the central nervous system in the acquired immunodeficiency syndrome. Hum Pathol 1987 ; 18 : 636-43.

22. Rance NE, McArthur JC, Cornblath DR, Landstrom DL, Griffin JW, Price DL. Gracile tract degeneration in patients with sensory neuropathy and AIDS. Neurology 1988 ; $38:$ 265-71.

23. Kim TS, Belman A, Dickson DW. Spinal cord pathology in pediatric AIDS. J Neuropathol Exp Neurol 1988; 47 : 303 (abstr.).

24. Gray F, Gherardi $R$, Baudrimont $M$, et al. Leukoencephalopathy with multinucleated giant cells containing HIV-like particles and multiple opportunistic cerebral infections in one patient with AIDS. Acta Neuropathol (Berl) 1987 ; $73: 99-104$.

25. Kleihues $P$, Lang W, Burger PC, et al. Progressive diffuse leukoencephalopathy in patients with acquired immune deficiency syndrome. Acta Neurapathol (Berl) 1985 ; 68 : chez des patients immunodéprimés d'autre origine [19], et l'absence de myélopathies vacuolaires chez les enfants et les nourrissons qui présentent habituellement des encéphalites à HIV pures [20] viennent contredire cette hypothèse.

D'autres lésions des voies longues intramédullaires ont été décrites. Rhodes [21] a rapporté un cas où une dégénérescence des voies pyramidales semblait secondaire à des lésions nécrotiques des capsules internes* contenant des cellules géantes multinucléées. Une dégénérescence incomplète des faisceaux de Goll**, chez quatre malades atteints de SIDA souffrant de paresthésies distales, a fait évoquer par Rance $e t$ al. [22] la possibilité d'une atteinte des ganglions spinaux lombaires par le HIV. Chez le nourrisson et le jeune enfant souffrant d'une infection sévère du SNC par le HIV, il n'a jamais été observé de myélopathie vacuolaire, mais l'atteinte médullaire n'est pas rare. Ainsi, dans une série de 15 cas rapportés par Kim et al. [23], 14 présentaient des signes de spasticité***. Dix de ces enfants avaient une pâleur myélinique des faisceaux pyramidaux correspondant, pour certains, à des lésions mixtes axonales et myéliniques évoquant une axonopathie et, pour d'autres, à une atteinte myélinique prédominante pouvant correspondre à un retard de myélinisation.

- Une imprégnation sidéro-calcique des parois vasculaires est fréquemment observée dans la substance blanche et dans les noyaux gris centraux, associée à l'encéphalite subaiguë. Certes, ces lésions sont peu spécifiques chez l'adulte, encore que, chez l'adulte jeune, elles ne soient pas si banales ; en revanche, leur présence constante chez les enfants est tout à fait remarquable. Un trouble du métabolisme phospho-calcique ne peut être formellement éliminé, mais celui-ci n'a jamais été démontré et le rôle d'une agression vasculaire par le

\footnotetext{
* Capsule interne: portion de la substance blanche hémisphérique séparant le thalamus en dehors des noyaux lenticulaires, en dedans et par où passe le faisceau pyramidal.

* Faisceau de Goll : colonne dorsale de la moelle épinière.

** Spasticité : hypertonie musculaire par exagération du réflexe monosynaptique.
}

HIV, comme on l'observe dans la rubéole congénitale, a été évoqué et semble confirmé par certaines observations pédiatriques.

\section{Autres lésions}

- Des lésions vasculaires sont possibles, mais elles sont rarement responsables du décès. Il peut s'agir d'hémorragies qui peuvent siéger à l'intérieur d'une tumeur ou dans une lésion de la substance blanche; elles peuvent aussi être la conséquence de troubles de la coagulation. Des lésions ischémiques peuvent être liées à une endocardite thrombotique non bactérienne, à une coagulopathie intravasculaire disséminée ou à une artérite spécifique.

- Des lésions pontiques* ont été signalées. Il peut s'agir de myélinolyse centrale du pont, conséquence d'une hyponatrémie prolongée, ou d'une leucoencéphalopathie pontine multifocale, semblable à celle observée chez les malades traités par radio et chimiothérapie.

- Enfin des lésions terminales d'anoxie-ischémie ou d'encéphalopathie hépatique ont été fréquemment notées.

\section{Des atteintes polymorphes et complexes}

Certains traits particuliers aux complications neuropathologiques du SIDA méritent d'être soulignés.

- L'incidence des lésions varie suivant de multiples facteurs. Suivant le terrain : il est remarquable que les enfants présentent exceptionnellement des infections opportunistes et ont très fréquemment une encéphalite subaiguë à CGM avec atrophie cérébrale et calcifications des noyaux gris, mais pas de myélopathie vacuolaire. Les hémophiles présentent le plus souvent des hémorragies et une encéphalopathie hépatique, et ont exceptionnellement une encéphalite à HIV et/ou des infections opportunistes. D'un pays à l'autre: la fréquence des différentes complications n'est pas la même en Europe, au Bré-

* Lésions pontiques : lésions du pont, région médiane du tronc cérébral. 
sil, sur la côte Est ou sur la côte Ouest des États-Unis.Dans le temps : ainsi la toxoplasmose cérébrale est de moins en moins fréquente, au moins à l'examen post-mortem, alors que les encéphalites virales (à HIV, CMV ou papovavirus) ont une incidence croissante.

- L'aspect neuropathologique des lésions est souvent atypique, trompeur, que ce soit du fait de l'immunodépression, ou de facteurs propres au SIDA.

- Enfin l'association de divers processus pathologiques est la règle ; successivement, mais aussi simultanément; dans le système nerveux et le reste de l'organisme, mais aussi à l'intérieur du système nerveux, au sein de la même lésion, voire au sein de la même cellule. Cette association semble assez particulière au SIDA et n'a été signalée que très rarement dans les immunodépressions d'autre origine [24].

L'intrication des lésions infectieuses et des CGM est particulièrement remarquable [7, 8]. Cette intrication de CGM avec d'autres infections opportunistes suggère deux possibilités : d'une part, la présence du HIV dans certaines régions du système nerveux central pourrait favoriser la survenue d'infections opportunistes dans cette région ; d'autre part, du fait de la présence diffuse du HIV dans le système nerveux central, certains des macrophages attirés par une autre lésion pourraient prendre l'aspect de cellules géantes multinucléées. Cette association très étroite entre les CGM et des inclusions virales de CMV ou de papovavirus a conduit Kleihues $e t$ al. [25] à se demander si les lésions diffuses de la substance blanche de la leucoencéphalopathie progressive diffuse, dont le mécanisme est peu clair, n'étaient pas dues à l'action synergique du HIV et d'un ou plusieurs autres virus.

Au total, l'aspect très variable et atypique des lésions, joint à l'association fréquente de divers processus pathologiques, rend difficile un diagnostic neuropathologique précis et doit rendre très prudent quant aux indications et aux interprétations des biopsies cérébrales à visée diagnostique. Ceci explique aussi la grande variabilité du tableau clinique et les difficultés rencontrées pour définir des entités anatomo-cliniques spécifiques

\section{Summary}

Central nervous system lesions in AIDS

The central nervous system has been involved in the majority (at least $75 \%$ ) of cases of AIDS examined post-mortem but the pathogenetic mechanisms involved are not well understood. The predominant pathological process is opportunistic infection secondary to the decrease of $T$ helper (T4) cells and includes toxoplasmosis, encephalitis due to cytomegalovirus and progressive multifocal leucoencephalopathy. Mycoses, mainly crytococcosis are relatively uncommon. Non-Hodgkin lymphomas could also be secondary to $T$-helper depletion; primary lymphomas are three times more common that secondary lymphoma spreading from other sites. Cerebral deposits of Kaposi sarcoma are rare and are probably metastatic. Lesions due to the direct infection of the central nervous system by the human immune deficiency virus (HIV) include subacute encephalitis and vacuolar myelopathy. The former is reported with increasing frequency and involves mainly the white matter in which multinucleated giant cells can be found. These are considered typical of AIDS and have been shown to contain HIV particles in their cytoplasm. These lesions are often atypical and very commonly combined making the diagnosis difficult.

\section{Fondation de physiopathologie professeur Lucien Dautrebande}

En date du 13 décembre 1988, a été remis, en présence de Sa Majesté la Reine, le prix de la Fondation Lucien Dautrebande au Professeur Godfraind pour ses recherches sur les antagonistes du calcium ; le prix du jeune chercheur a été remis au Docteur Henquin pour son travail sur le contrôle de la sécrétion de l'insuline par les îlots de Langerhmans. Les deux lauréats sont attachés à l'Université Catholique de Louvain.

Précédemment, ce prix avait été attribué successivement au Professeur Durrer d'Amsterdam en 1973, au Professeur Gajdusek de Bettesda en 1976 (celui-ci obtint ultérieurement le Prix Nobel), au Professeur Desmedt de Bruxelles en 1979, aux Professeurs Hugues et Kosterlitz de Londres et d'Aberdeen en 1982 et au Professeur Baxter de San Francisco en 1985.

Le prochain prix d'un montant d'environ $4000000 \mathrm{~F}$ sera décerné en 1991.

Le règlement du Prix peut être obtenu en écrivant au Président de la Fondation de Physiopathologie Professeur Lucien Dautrebande:

Docteur Stalport, "Maison Batta», Avenue Batta 3, B-5200 Huy, Belgique.

Les candidatures doivent lui être adressées auant le 31 décembre 1990. 